\title{
Reduction in albuminuria predicts a beneficial effect on diminishing the progression of human diabetic nephropathy during antihypertensive treatment
}

\author{
P. Rossing, E.Hommel, U.M.Smidt, H.-H. Parving \\ Steno Diabetes Center, Gentofte, Denmark
}

Summary Diabetic nephropathy is the main cause of increased mortality and morbidity in IDDM patients. The effect of antihypertensive treatment on the progression of the nephropathy is highly variable. The aim of this study was to evaluate putative predictors of the progression in diabetic nephropathy during long-term antihypertensive treatment. Eighteen hypertensive IDDM patients with diabetic nephropathy, who had not been treated previously, were followed during 3 years of treatment with captopril and frusemide or bendrofluazide. Glomerular filtration rate, arterial blood pressure, albuminuria and adjusted albuminuria were used as putative predictors of rate of decline in glomerular filtration. Fall rate in glomerular filtration rate was $4.6(4.0) \mathrm{ml} \cdot \mathrm{min}^{-1} \cdot$ year $^{-1}$ (mean (SD)) during treatment. Relative change in albuminuria (ratio of first year of treatment/baseline) and albuminuria during first year of treatment were significantly correlated to fall rate in glomerular filtration rate during 3 years of treatment $(r=0.73, p<0.001)$ and $(r=0.60, p<0.01)$, respectively. Arterial blood pressure and glomerular filtration rate measured at baseline, during first year of treatment or relative changes in these variables did not correlate with fall rate in glomerular filtration rate during 3 years of treatment. Haemoglobin $A_{1 c}$, serum-cholesterol, protein intake and sodium excretion remained unchanged during treatment, and were not correlated with loss of kidney function. Reduction in albuminuria during captopril treatment predicts an attenuated rate of decline in glomerular filtration rate in early diabetic nephropathy (glomerular filtration rate $>70 \mathrm{ml}$. $\mathrm{min}^{-1} \cdot 1.73 \mathrm{~m}^{-2}$ ). The finding suggests a clinical application in monitoring the efficacy of antihypertensive treatment in early diabetic nephropathy. [Diabetologia (1994) 37:511-516]

Key words Diabetic nephropathy, albuminuria, antihypertensive treatment, glomerular filtration rate.
Diabetic nephropathy develops in $30-40 \%$ of IDDM patients $[1,2]$, and nephropathy is the main cause of increased morbidity and mortality in these patients [1]. Diabetic nephropathy is a clinical syndrome characterized by persistent albuminuria, a relentless decline in glomerular filtration rate, and raised arterial blood pressure [3]. Human and animal studies have demonstrated a correlation between elevation in arterial

Received: 21 September 1993 and in revised form: 3 December 1993

Corresponding author: Dr. P. Rossing, Steno Diabetes Center, Niels Steensens Vej 2, DK-2820 Gentofte, Denmark

Abbreviations: IDDM, Insulin-dependent diabetes mellitus blood pressure and development and progression of diabetic glomerulopathy [4-10]. Several studies [1117] have demonstrated that the rate of decline in glomerular filtration rate can be reduced by antihypertensive treatment. However the beneficial effect of antihypertensive treatment on the progression of the diabetic nephropathy is highly variable. From a clinical point of view, identification of predictors of the longterm effect on kidney function of a recently initiated treatment modality would be important. Patients, for whom poor prognosis is predicted, could then be given intensified treatment. Preliminary analysis of kidney function data in diabetic nephropathy has led us to suggest, that a decrease in albuminuria shortly after onset of antihypertensive treatment predicts a low rate of decline in glomerular filtration rate [18]. 
Table 1. Clinical data of 18 IDDM patients with diabetic nephropathy

\begin{tabular}{ll}
\hline Sex (female/male) & $4 / 14$ \\
Age (years) & $33(8)$ \\
Duration of diabetes & $21(7)$ \\
Retinopathy (Nil/background/proliferative) & $0 / 7 / 11$ \\
Insulin dose $\left(\mathrm{IU} \cdot \mathrm{kg}^{-1} \cdot\right.$ day $\left.^{-1}\right)$ & $0.60(0.15)$ \\
Body mass index $\left(\mathrm{kg} / \mathrm{m}^{2}\right)$ & $23.4(1.7)$ \\
\hline
\end{tabular}

Values are mean (SD)

In an attempt to evaluate this concept and thereby obtain a clinical guideline for the future loss of kidney function after onset of antihypertensive treatment, we analysed data from a prospective study of the effect on kidney function of antihypertensive treatment with an angiotensin converting enzyme inhibitor in IDDM patients with early diabetic nephropathy (glomerular filtration rate $>70 \mathrm{ml} \cdot \mathrm{min}^{-1} \cdot 1.73 \mathrm{~m}^{-2}$ ) [16]. The following putative predictors were evaluated: arterial blood pressure, glomerular filtration rate, urinary albumin excretion, adjusted albumin excretion (albumin excretion/glomerular filtration rate).

\section{Subjects and methods}

\section{Patients}

Eighteen patients with IDDM and persistent albuminuria attending Steno Diabetes Center were included in this study (Table 1). The patients comprise the entire treatment group in a non-randomized controlled trial of matched hypertensive IDDM patients with nephropathy treated with captopril and frusemide or bendrofluazide [16]. All patients fulfilled the following inclusion criteria: age under 50 , persistent albuminuria ( $>300 \mathrm{mg} /$ day), a serum creatinine concentration less than $120 \mu \mathrm{mol} / \mathrm{l}$, no oedema, diagnosis of hypertension, but not receiving antihypertensive treatment (including diuretics), development of diabetes before the age of 31 , and not blind.

Antihypertensive treatment with captopril ( 37.5 to $100 \mathrm{mg}$ daily) alone (one patient) or in combination with frusemide (mean $98.0 \mathrm{mg}$ daily, 10 patients) or bendrofluazide (mean $4.0 \mathrm{mg}$ daily, seven patients) was started after the baseline investigation. The aim was to achieve a stable reduction in diastolic blood pressure to below $90 \mathrm{~mm} \mathrm{Hg}$ and of at least $10 \mathrm{~mm} \mathrm{Hg}$ in mean arterial blood pressure (diastolic pressure plus one-third of the pulse amplitude), or both.

All patients were placed on insulin therapy from the time of diagnosis, and all received at least two daily injections of highly purified porcine insulin. They had a normal diabetic diet containing $45-55 \%$ carbohydrate, $30-35 \%$ fat, and $15-20 \%$ protein throughout the study. None of the patients had their intake of protein or salt restricted. Hypertension was diagnosed if the mean of the last three measurements performed in the out-patient clinic before the start of the study was equal to or greater than $160 / 90 \mathrm{~mm} \mathrm{Hg}$. Nephropathy was diagnosed clinically if there was persistent albuminuria, diabetic retinopathy, diabetes of more than 10 years duration, and no clinical or laboratory evidence of kidney or renal-tract disease other than diabetic nephropathy [11]. The study was approved by the local ethical committee, and the investigated patients all gave their informed consent.

\section{Methods}

All investigations were carried out on one day between 08.30 and 13.00 hours. Patients had their normal breakfast and morning dose of insulin before the investigations, during which they rested supine and stood up only to pass urine. They drank $150-200 \mathrm{ml}$ tap water per $h$ during the study. The investigations were carried out 5 to 7 times during 31-36 (mean 34) months. The patients were studied at baseline, after 3 months, and then at 6 -month intervals.

The glomerular filtration rate was measured after a single intravenous injection of edetic acid labelled with $3.7 \mathrm{MBq}$ sodium chromate- 51 at 09.00 hours, by determining the radioactivity in venous blood samples taken from the other $\operatorname{arm} 180,200,220$, and $240 \mathrm{~min}$ after the injection $[19,20]$. The results were standardized for $1.73 \mathrm{~m}^{2}$ body surface area, using the patients surface area at the start of the study. The mean coefficient of variation in the glomerular filtration rate of each patient from day to day was $4 \%$.

Urinary albumin excretion was measured during the 4-h clearance period by radioimmunoassay [21]. The assay had a sensitivity of $0.5 \mathrm{mg} / \mathrm{l}$ and a coefficient of variation between assays of $9 \%$. The patients collected urine for one 24 -h period before the investigations for measuring the excretion of sodium and urea. The urea excretion was used to calculate protein intake from the nitrogen content of the urea and an estimated value of non-urea nitrogen of $31 \mathrm{mg} \cdot \mathrm{kg}^{-1} \cdot \mathrm{day}^{-1}$ [22]. (Assuming a constant nitrogen balance, nitrogen intake $=$ nitrogen content of urinary urea plus nonurea nitrogen; protein intake $(g /$ day $)=$ nitrogen intake $\times 6.25)[23]$.

Blood pressure was measured after $10 \mathrm{~min}$ or more rest with the patient in the supine position with a standard clinical mercury sphygmomanometer (cuff $25 \times 12 \mathrm{~cm}$ ) on the right arm. Blood pressure was measured two times between 09.00 and 10.00 hours and between 12.00 and 13.00 hours during the study, and two times at each visit to the out-patient clinic. Diastolic blood pressure was recorded as the disappearance of the Korotkoff sounds (phase V). Serum cholesterol concentration measured by conventional laboratory technique and stable haemoglobin $A_{1 c}$ concentration (normal range $4.1-6.1 \%$ of total haemoglobin) were measured during each investigation. All patients visited the clinic every 2 to 4 months during the investigation. At each visit the postprandial blood glucose concentration was measured along with urinary glucose excretion, blood pressure and body weight, and the dose of insulin and antihypertensive treatment were adjusted. The following putative predictors of rate of decline in glomerular filtration rate during antihypertensive treatment were evaluated: arterial blood pressure, glomerular filtration rate, albuminuria, adjusted albuminuria (albuminuria/glomerular filtration rate), at baseline, and for the first year during antihypertensive treatment, respectively. The relative changes in these variables after onset of antihypertensive treatment were also evaluated as predictors (((during-baseline)/baseline) $* 100 \%)$. Serum concentration of haemoglobin $A_{1 c}$, cholesterol, protein intake and sodium excretion during the 3 years of treatment were determined as possible confounders. Rate of decline in glomerular filtration rate was calculated for the whole treatment period.

\section{Statistical analysis}

Values are given as mean (SD). Values for urinary albumin excretion rate and adjusted albuminuria were logarithmically transformed before analysis because of their positively skewed distribution, median (range) are given. Wilcoxon's non-parametric test was used for paired comparison. Kruskall-Wallis test of variance was used for comparison between three groups, if a difference was found, the Mann-Whitney U-test was used for com- 
Table 2. Arterial blood pressure, albuminuria, glomerular filtration rate, adjusted albuminuria (albuminuria/glomerular filtration rate) at baseline, and the first year during antihypertensive treatment, and the relative changes in these variables $((($ duringbaseline)/baseline $) \cdot 100 \%)(n=18)$

\begin{tabular}{|c|c|c|c|}
\hline & Baseline & $\begin{array}{l}\text { First year during } \\
\text { treatment }\end{array}$ & $\begin{array}{l}\text { Relative change (\%) } \\
\text { (mean(SD)) }\end{array}$ \\
\hline Blood pressure $(\mathrm{mm} \mathrm{Hg})^{a}$ & $147 / 93(11 / 5)$ & $131 / 84(11 / 6)^{c}$ & $-10 /-10(6 / 5)$ \\
\hline Albuminuria $(\mu \mathrm{g} / \mathrm{min})^{\mathrm{b}}$ & $1295(168-2946)$ & $646(71-2216)^{c}$ & $-40(35)$ \\
\hline Glomerular filtration rate $\left(\mathrm{ml} \cdot \mathrm{min}^{-1} \cdot 1.73 \mathrm{~m}^{-2}\right)^{\mathrm{a}}$ & $98(22)$ & $91(26)^{d}$ & $-9(8)$ \\
\hline Adjusted albuminuria $\left(\mu \mathrm{g} \cdot \mathrm{ml}^{-1} \cdot 1.73 \mathrm{~m}^{-2}\right)^{\mathrm{b}}$ & $12(2-34)$ & $8(1-25)^{d}$ & $-35(37)$ \\
\hline
\end{tabular}

${ }^{\mathrm{a}}$ Mean (SD), ${ }^{\mathrm{b}}$ median (range);

${ }^{\mathrm{c}} p<0.001 ;{ }^{\mathrm{d}} p<0.01$ vs baseline

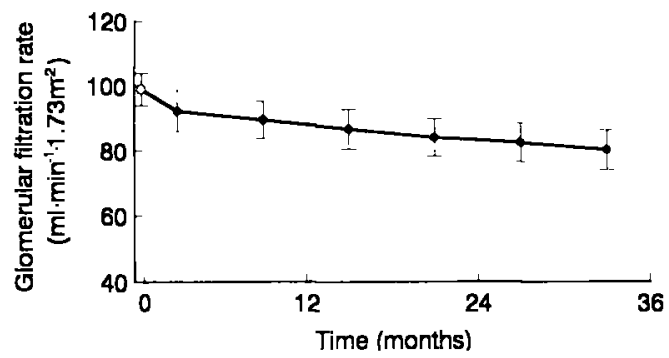

Fig. 1. Mean course of glomerular filtration rate in 18 IDDM patients with diabetic nephropathy at baseline $\left(^{\circ}\right.$ ) and during antihypertensive treatment $(\bullet)$. Bars are SEM

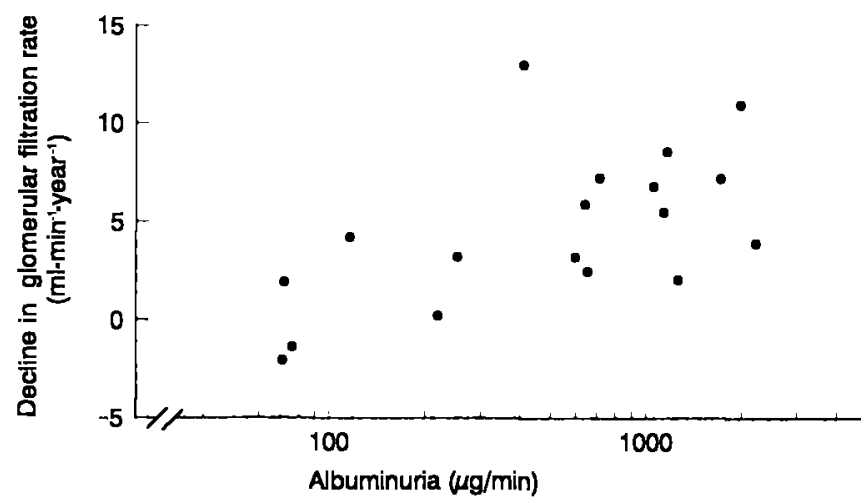

Fig. 2. Correlation between albuminuria (log scale) and rate of decline in glomerular filtration rate $(r=0.60 p<0.01)$. Albuminuria was measured during the first year after start of antihypertensive treatment. Glomerular filtration rate was measured during 3 years of antihypertensive treatment in 18 IDDM patients with diabetic nephropathy

parison between two groups. Linear regression analysis (least squares method) was used to determine the rate of decline in glomerular filtration rate for each patient, using all measured values of glomerular filtration rate from the treatment period. Univariate linear regression analysis was used to analyse data for correlations between the above-mentioned putative predictors and rate of decline in glomerular filtration rate. Forward stepwise multiple linear regression analysis was made with the putative predictors as independent variables and rate of decline in glomerular filtration rate as the dependent variable. These analyses were made using absolute values of putative predictors measured at baseline or during the first year of treatment, or with the relative changes in the putative predictors. All calculations were made using Statgraphics (STSC, Rockville, Md., USA). A p value of less than 0.05 was considered significant (two-tailed).
Results

Arterial blood pressure, albuminuria, glomerular filtration rate and adjusted albuminuria decreased significantly after onset of antihypertensive treatment (Table 2). The blood pressure was reduced after 3 months of antihypertensive treatment and was maintained at $136 / 85(8 / 5) \mathrm{mm} \mathrm{Hg}$ during the 3 years of treatment. During the entire treatment period haemoglobin $\mathrm{A}_{1 \mathrm{c}}$ (normal range 4.1 to $6.1 \%$ ) and serum cholesterol concentration were $9.3(0.8) \%$ and $6.5(0.8)$, respectively, both were unchanged compared to baseline. Average protein intake was $1.1(0.3) \mathrm{g} \cdot \mathrm{kg}^{-1} \cdot \mathrm{day}^{-1}$ and urinary sodium excretion was 154 (63) mmol/day. The rate of decline in glomerular filtration rate was 4.6 (4.2) $\mathrm{ml} \cdot \mathrm{min}^{-1} \cdot$ year $^{-1}$, ranging from -2.1 to 13.0 (Fig. 1).

Forward stepwise multiple linear regression analysis with rate of decline in glomerular filtration rate as the dependent variable and the absolute levels of the putative predictors (arterial blood pressure, albuminuria, glomerular filtration rate and adjusted albuminuria) measured at baseline or the first year during treatment, respectively, as independent variables, showed only a significant correlation with albumin excretion during the first year of treatment $(r=0.60$, $p<0.01$, Fig. 2).

Furthermore the relative change in the putative predictors was calculated using first year during treatment vs baseline. In a stepwise linear regression analysis there was a significant correlation between the loss of kidney function and relative change in albuminuria $(r=0.73, p<0.001$, Fig. 3). In a univariate regression analysis there was a significant correlation between relative change in adjusted albuminuria and rate of decline in glomerular filtration rate $(r=0.72, p<0.001)$. The patients were divided into tertiles based on relative change in albuminuria (Table 3 ), the group with the largest decrease in albuminuria had a rate of decline in glomerular filtration rate of $1.5 \mathrm{ml} \cdot \mathrm{min}^{-1} \cdot \mathrm{year}^{-1}$, while the rate of decline was $8.3 \mathrm{ml} \cdot \mathrm{min}^{-1} \cdot \mathrm{year}^{-1}$ in the group with stable or increasing albuminuria $(p=0.01)$. There was no significant correlation between the relative change in systolic or diastolic blood pressure and the relative change in albuminuria or adjusted albuminuria (during/baseline), respectively. 
To evaluate the predictive value of the earliest demonstrable changes, we repeated the above-described stepwise regression analyses using baseline values and the first measurement during treatment, which were performed after 3 months of treatment. There was a significant correlation between the absolute values of albuminuria after 3 months and rate of decline in glomerular filtration rate $(r=0.49, p<0.05)$ and between the relative change in adjusted albuminuria and the loss of kidney function $(r=0.48, p<0.05)$.

In a stepwise linear regression analysis with rate of decline in glomerular filtration rate as the dependent variable and serum concentrations of haemoglobin $A_{1}$, cholesterol, protein intake and sodium excretion, as independent variables, (averages of values obtained during the 3-year treatment period were used), there was no significant correlation. These variables remained unchanged during the study period.

\section{Discussion}

Our study suggests that low levels of albuminuria measured shortly after the start of antihypertensive treatment with captopril and diuretics predicts an attenuated fall in glomerular filtration rate in IDDM patients with early diabetic nephropathy (glomerular fil-

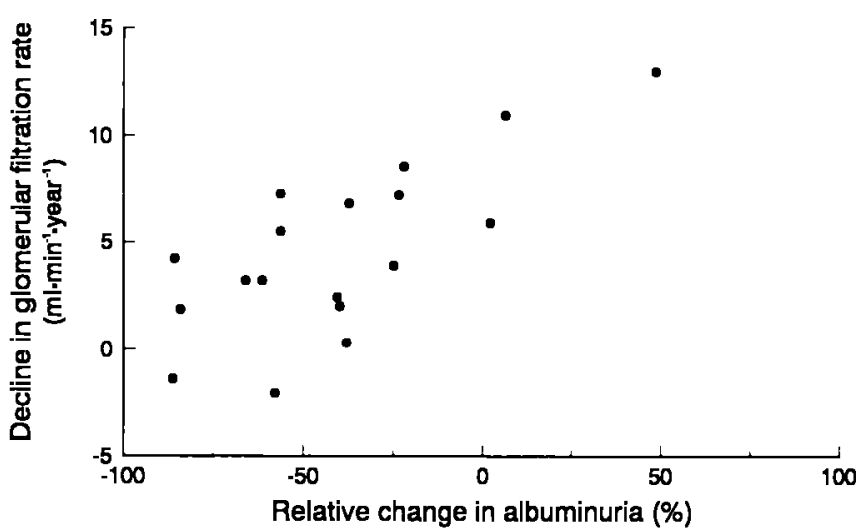

Fig.3. Correlation between relative change in albuminuria and decline in glomerular filtration rate $(r=0.73 p<0.001)$. Albuminuria was measured at baseline and during the first year after start of antihypertensive treatment, respectively. Glomerular filtration rate was measured during 3 years of antihypertensive treatment in 18 IDDM patients with diabetic nephropathy tration rate $>70 \mathrm{ml} \cdot \mathrm{min}^{-1} \cdot 1.73 \mathrm{~m}^{-2}$ ). This guideline is further improved if pretreatment values are known, since the relative change in albuminuria or adjusted albuminuria are more closely correlated with the future fall in glomerular filtration rate. We have demonstrated a correlation between initial measurements of albuminuria (surrogate end-point) and long-term preservation of glomerular filtration rate (principal endpoint) in diabetic nephropathy. Our study did not support the suggestion that arterial blood pressure and glomerular filtration rate measured initially during antihypertensive treatment can be used as a clinical guideline for the future loss of kidney function, but numbers were small. Other putative confounders such as metabolic control, serum cholesterol, protein intake and sodium excretion could not explain the wide range in fall rate in glomerular filtration rate during antihypertensive treatment.

Furthermore, in a preliminary analysis, we have demonstrated a correlation between relative change in adjusted albuminuria and rate of decline in glomerular filtration rate in 20 IDDM patients with diabetic nephropathy (glomerular filtration rate $>37 \mathrm{ml} \cdot \mathrm{min}^{-1}$. $1.73 \mathrm{~m}^{-2}$ ) treated with metoprolol and frusemide [18]. Recently Apperloo et al. [24] have demonstrated that the reduction in proteinuria after 12 weeks of therapy correlated with the long-term rate of decline in glomerular filtration rate in 30 proteinuric non-diabetic patients (glomerular filtration rate $>30 \mathrm{ml} \cdot \mathrm{min}^{-1}$. $1.73 \mathrm{~m}^{-2}$ ) treated for 2 years with atenolol or enalapril in a double-blind trial.

Treatment which successfully has reduced the progression in diabetic kidney disease both experimentally $[8,9,25]$ and in clinical trials $[12,13,16,17,26]$ has always been associated with a reduction in proteinuria during the intervention period. The association between albuminuria and progression of diabetic nephropathy, suggests that measurements of the initial antiproteinuric effect may provide a tool for rapid screening of the potential renoprotective effect of new drugs and non-pharmacological treatment regimens. Further evaluation of this concept are urgently needed in diabetic and other glomerulopathies. The impact of antihypertensive treatment on albumin excretion must reflect functional or structural factors contributing directly or indirectly to the loss of kidney function. As the antiproteinuric effect is observed shortly after treat-

Table 3. Impact of relative change in albuminuria (((first year during treatment-baseline)/baseline) $100 \%)$ on the progression of diabetic nephropathy in 18 IDDM patients

\begin{tabular}{|c|c|c|c|}
\hline & \multicolumn{3}{|l|}{ Tertiles } \\
\hline & Largest reduction $(n=6)$ & Intermediate $(n=6)$ & No reduction $(n=6)$ \\
\hline Change in albuminuria (\%) (range) & -86 to -58 & -57 to -26 & -25 to 49 \\
\hline $\begin{array}{l}\text { Rate of decline in glomerular } \\
\text { filtration rate }\left(\mathrm{ml} \cdot \min ^{-1} \cdot \text { year }^{-1}\right)^{a}\end{array}$ & $1.5(-1.2$ to 4.2$)$ & $4.0(1.0$ to 7.1$)$ & $8.3(4.8$ to 11.7$) b$ \\
\hline
\end{tabular}

${ }^{\mathrm{a}}$ Mean (95\% confidence interval); ${ }^{\mathrm{b}} p<0.01$ Largest vs no reduction 
ment is initiated, it is most likely caused by functional changes, since structural lesions would probably not regress that quickly. The observed antiproteinuric effect of captopril and frusemide/bendrofluazide may be due either to diminished glomerular capillary hydraulic pressure or to enhanced intrinsic selectivity of the glomerular capillary barrier wall, or to both. Increased glomerular capillary hydraulic pressure has been shown to accelerate the development of diabetic glomerulopathy in experimental animals [9], while antihypertensive medication reduces intraglomerular pressure, albuminuria and the severity of diabetic glomerular lesions [25]. The antiproteinuric effect is mainly/solely present if the reduction in systemic blood pressure leads to a decrease in intraglomerular pressure $[25,27]$. Inhibition of angiotensin converting enzyme prevents the action of angiotensin II on the efferent arteriole, thereby diminishing the intraglomerular pressure irrespective of the effect on systemic hypertension.

The size and charge selectivity of the glomerular capillary filtration barrier is impaired in patients with diabetic nephropathy compared to patients with normo- and microalbuminuria [28-30]. Scandling et al. [31] have shown in diabetic as well as other proteinuric nephropathies that there is a reciprocal relationship between the fraction of large pores (resposible for proteinuria) and pore density (ultrafiltration capacity). This is consistent with our prediction if the same membrane change that lowers the glomerular permeability to albumin (fraction of large pores) also increases the filtration capacity (pore density). Antihypertensive treatment with an angiotensin converting enzyme inhibitor has been shown to improve both size and charge selective properties [32,33].

Remuzzi et al. [34] has suggested that proteinuria is not simply a marker of the extent of glomerular damage, but that proteinuria per se may contribute to the kidney lesions. The potential mechanisms by which proteinuria may itself promote damage to mesangial, epithelial and tubular cell function has been critically reviewed [34]. Our finding of a correlation between albuminuria and rate of progression in kidney function is compatible with the concept that albuminuria per se may be harmful to the kidney. We have previously demonstrated a correlation between the magnitude of albuminuria and the rate of decline in glomerular filtration rate in an observational study of the natural history of diabetic nephropathy [35].

Marked proteinuria causes dyslipidaemia with hypercholesterolaemia and hypertriglyceridaemia with increasing LDL and VLDL [36, 37]. Hyperlipidaemia has been shown to play a role in initiation and progression of glomerular injury in animal models [38, 39]. A positive correlation has been found between serum cholesterol and rate of decline in glomerular filtration rate in IDDM patients with diabetic nephropathy [17] and in proteinuric non-diabetic patients [24].
Animal studies have demonstrated a reduced dyslipidaemia after reduction in albuminuria with antihypertensive treatment [40]. In our study we found no change in serum cholesterol after onset of treatment and no correlation was found between cholesterol and loss of kidney function.

In conclusion our study suggests that a decrease in albuminuria shortly after onset of antihypertensive treatment with an angiotensin converting enzyme inhibitor predicts a low rate of decline in glomerular filtration rate in the early stages of diabetic nephropathy. The finding suggests a clinical application to monitor the efficacy of antihypertensive treatment in early diabetic nephropathy. The validity of this suggestion in more advanced diabetic nephropathy (glomerular filtration rate $<70 \mathrm{ml} \cdot \min ^{-1} \cdot 1.73 \mathrm{~m}^{-2}$ ) remains to be evaluated.

\section{References}

1. Andersen AR, Christiansen JS, Andersen JK, Kreiner S, Deckert T (1983) Diabetic nephropathy in type 1 (insulin-dependent) diabetes: an epidemiological study. Diabetologia 25: 496-501

2. Krolewski AS, Warram JH, Christlieb AR, Busick EJ, Kahn CR (1985) The changing natural history of nephropathy in type 1 diabetes. Am J Med 78: 785-794

3. Deckert T, Poulsen JE, Larsen M (1978) Prognosis of diabetics with diabetes onset before the age of thirtyone. I.Survival, causes of death and complications. Diabetologia 14: 363-370

4. Mogensen CE (1976) Progression of nephropathy in longterm diabetics with proteinuria and effect of initial antihypertensive treatment. Scand J Clin Lab Invest 36: 383-388

5. Feldt-Rasmussen B, Mathiesen ER, Jensen T, Lauritzen T, Deckert T (1991) Effect of improved metabolic control on loss of kidney function in type 1 (insulin-dependent) diabetic patients: an update of the Steno studies. Diabetologia 34: $164-170$

6. Klahr S (1989) The modification of diet in renal disease study. N Engl J Med 320: 864-866

7. Steffes MW, Brown DM, Mauer SM (1979) The development, enhancement, and reversal of the secondary complications of diabetes mellitus. Hum Pathol 10: 293-299

8. Brenner BM (1983) Hemodynamically mediated glomerular injury and the progressive nature of kidney disease. Kidney Int 23: 647-655

9. Hostetter TH, Rennke HG, Brenner BM (1982) The case for intrarenal hypertension in the initiation and progression of diabetic and other glomerulopathies. Am J Med 72: 375-380

10. Steffes MW, Brown DM, Mauer SM (1978) Diabetic glomerulopathy following unilateral nephrectomy in the rat. Diabetes 27: 35-41

11. Parving H-H, Andersen AR, Smidt UM, Svendsen PAa (1983) Early aggressive antihypertensive treatment reduces rate of decline in kidney function in diabetic nephropathy. Lancet I: $1175-1179$

12. Mogensen CE (1982) Long-term antihypertensive treatment inhibiting progression of diabetic nephropathy. BMJ 285 : $685-688$

13. Parving $\mathrm{H}-\mathrm{H}$, Andersen AR, Smidt UM, Hommel E, Mathiesen ER, Svendsen PAa (1987) Effect of antihypertensive treatment on kidney function in diabetic nephropathy. BMJ 294: 1443-1447 
14. Parving H-H, Hommel E (1989) Prognosis in diabetic nephropathy. BMJ 299: 230-233

15. Mathiesen ER, Borch-Johnsen K, Jensen DV, Deckert T (1989) Improved survival in patients with diabetic nephropathy. Diabetologia 32: 884-886

16. Parving H-H, Hommel E, Smidt UM (1988) Protection of kidney function and decrease in albuminuria by captopril in insulin dependent diabetics with nephropathy. BMJ 297: 1086-1091

17. Björck S, Mulec H, Johnsen SAa, Nordén G, Aurell M(1992) Renal protective effect of enalapril in diabetic nephropathy. BMJ 304: 339-343

18. Rossing P, Hommel E, Smidt UM, Parving H-H (1992) Reduction in albuminuria predicts a beneficial effect on progression in diabetic nephropathy during antihypertensive treatment. Diabetologia 35 [Suppl 1]: A147(Abstract)

19. Bröchner-Mortensen J (1972) A simple method for the determination of glomerular filtration rate. Scand J Clin Lab Invest 30: 271-274

20. Bröchner-Mortensen J, Rödbro P (1976) Selection of routine method for determination of glomerular filtration rate in adult patients. Scand J Clin Lab Invest 36: 35-45

21. Miles DW, Mogensen CE, Gundersen HJG (1970) Radioimmunoassay for urinary albumin using a single antibody. Scand J Clin Lab Invest 26: 5-11

22. Maroni BJ, Steinman TI, Mitch WE (1985) A method for estimating nitrogen intake of patients with chronic renal failure. Kidney Int 27: 58-65

23. Isaksson $B$ (1980) Urinary nitrogen output as a validity test in dietary surveys. Am J Clin Nutr 33:4-5

24. Apperloo AJ, de Zeeuw D, de Jong PE (1992) The initial antiproteinuric effect of antihypertensive treatment predicts the effect of the treatment on long-term renal function outcome. J Am Soc Nephrol 3: 279(Abstract)

25. Anderson S, Rennke HG, Garcia DL, Brenner BM (1989) Short and long term effects of antihypertensive therapy in the diabetic rat. Kidney Int 36: 526-536

26. Walker JD, Bending JJ, Dodds RA et al. (1989) Restriction of dietary protein and progression of renal failure in diabetic nephropathy. Lancet II: $1411-1415$

27. Anderson S, Rennke HG, Brenner BM (1986) Therapeutic advantage of converting enzyme inhibitors in arresting progressive renal disease associated with systemic hypertension in the rat. J Clin Invest 77: 1993-2000
28. Viberti GC, Mackintosh D, Keen H (1983) Determinants of the penetration of proteins through the glomerular barrier in insulin-dependent diabetes mellitus. Diabetes 32:92-95

29. Deckert T, Feldt-Rasmussen B, Djurup R, Deckert M (1988) Glomerular size and charge selectivity in insulin-dependent diabetes mellitus. Kidney Int 33: 100-106

30. Tomlanovich S, Deen WM, Jones HW III, Schwartz HC, Myers BD (1987) Functional nature of glomerular injury in progressive diabetic glomerulopathy. Diabetes 36: 556-565

31. Scandling JD, Black VM, Deen WM, Myers BD (1992) Glomerular permselectivity in healthy and nephrotic humans. Adv Nephrol 21: 159-176

32. Morelli E, Loon N, Meyer T, Peters W, Myers BD (1990) Effects of converting-enzyme inhibition on barrier function in diabetic glomerulopathy. Diabetes 39: 76-82

33. Reddi AS, Ramamurthi R, Miller M, Dhuper S, Lasker N (1991) Enalapril improves albuminuria by preventing glomerular loss of heparan sulfate in diabetic rats. Biochem Med Metab Biol 45: 119-131

34. Remuzzi G, Bertani T (1990) Is glomerulosclerosis a consequence of altered glomerular permeability to macromolecules? Kidney Int 38: 384-394

35. Rossing P, Hommel E, Smidt UM, Parving H-H (1993) Impact of arterial blood pressure and albuminuria on the progression of diabetic nephropathy in insulin-dependent diabetic patients. Diabetes 42: 715-719

36. Moorhead JF, El-Nahas M, Chan MK, Varghese Z (1982) Lipid nephrotoxicity in chronic progressive glomerular and tubulo-interstitial disease. Lancet II: 1309-1311

37. Winocour PH, Durrington PN, Ishola $\mathrm{M}$, Anderson DC, Cohen $\mathrm{H}$ (1987) Influence of proteinuria on vascular disease, blood pressure, and lipoproteins in insulin dependent diabetes mellitus. BMJ 294: 1648-1651

38. Kasiske BL, O'Donnell MP, Schmitz PG, Kim Y, Keane WF (1990) Renal injury of diet-induced hypercholesterolemia in rats. Kidney Int 37: 880-891

39. Kasiske BL, O’Donnell MP, Cleary MP, Keane WF (1988) Treatment of hyperlipidemia reduces glomerular injury in obese Zucker rats. Kidney Int 33: 667-672

40. Kaysen GA, Davies RW (1990) Reduction in proteinuria attenuates hyperlipidemia in the nephrotic syndrome. J Am Soc Nephrol 1: S75-S79 